\title{
Magnetically Levitated Plasma Proteins
}

\author{
Ali Akbar Ashkarran†, Kenneth S. Suslick§, and Morteza Mahmoudi ${ }^{*}$ \\ †Precision Health Program, Michigan State University, East Lansing, Michigan 48824, United States \\ §Department of Chemistry, University of Illinois at Urbana-Champaign, Urbana, Illinois 61801, United States
}

The SI contains all supporting figures that are mentioned in the main text.

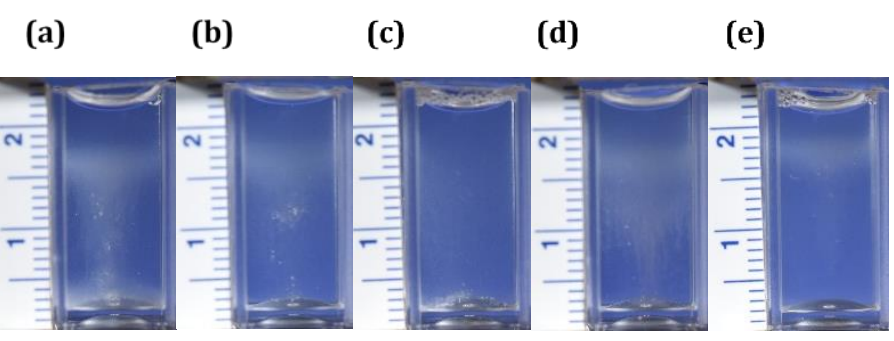

Figure S1: Levitation pattern of human plasma proteins in (a) $0.25 \mathrm{M} \mathrm{GdCl}_{3}$ and (b) $0.25 \mathrm{M} \mathrm{GV}$ solutions in DI water and levitation patterns of human plasma proteins in (c) $0.1 \mathrm{M}$, (d) $0.25 \mathrm{M}$ and (e) $0.5 \mathrm{M}$ GV solution in PBS.

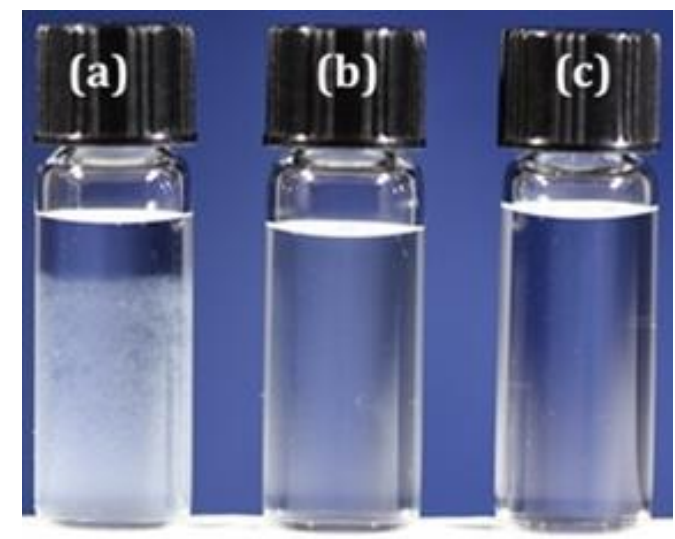

Figure S2: Photographs of (a) $1 \mathrm{M} \mathrm{GdCl}_{3}$ in PBS, (b) $2 \mathrm{M}$ gadovist in PBS and (c) $2 \mathrm{M} \mathrm{GdCl}_{3}$ in DI water. As is clear from the images, the solubility of $\mathrm{GdCl}_{3}$ in PBS even in lower concentrations is very low. This is while the solubility is much better in DI water even in higher concentration. Also, solubility of GV in PBS is quite high and there is no unsolved salt in the final solution. 


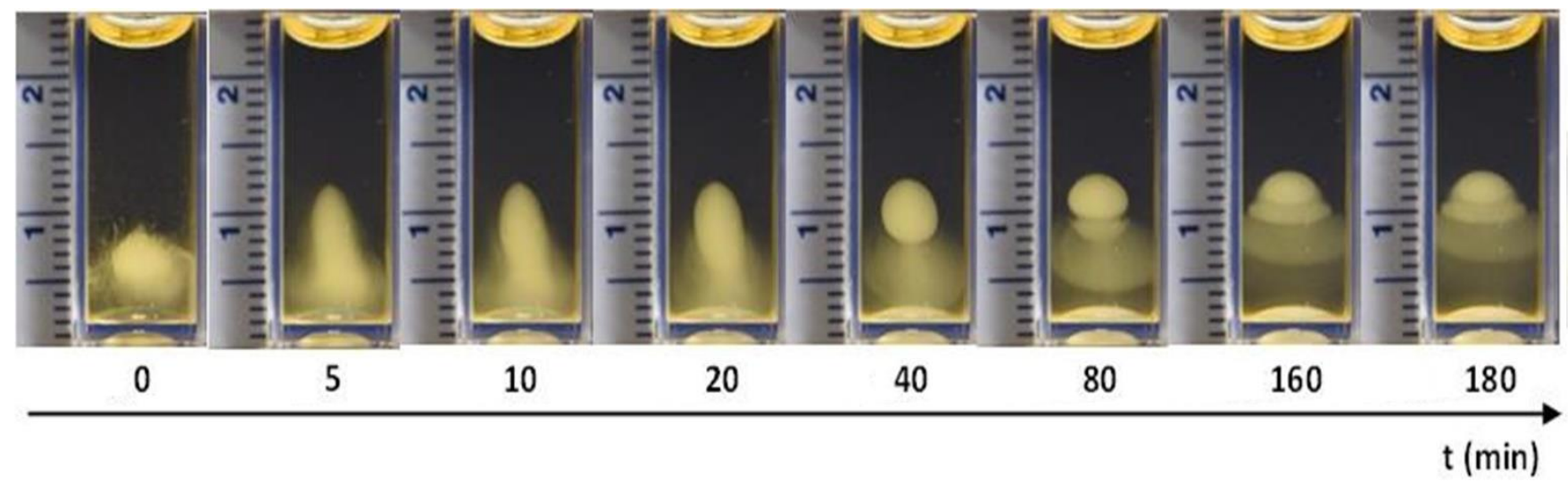

Figure S3: Photographs of the formation of ellipsoidal patterns from levitated plasma proteins over time in the MagLev system (0.06 $\mathrm{mg} / \mathrm{ml}$ concentration of SPIONs).

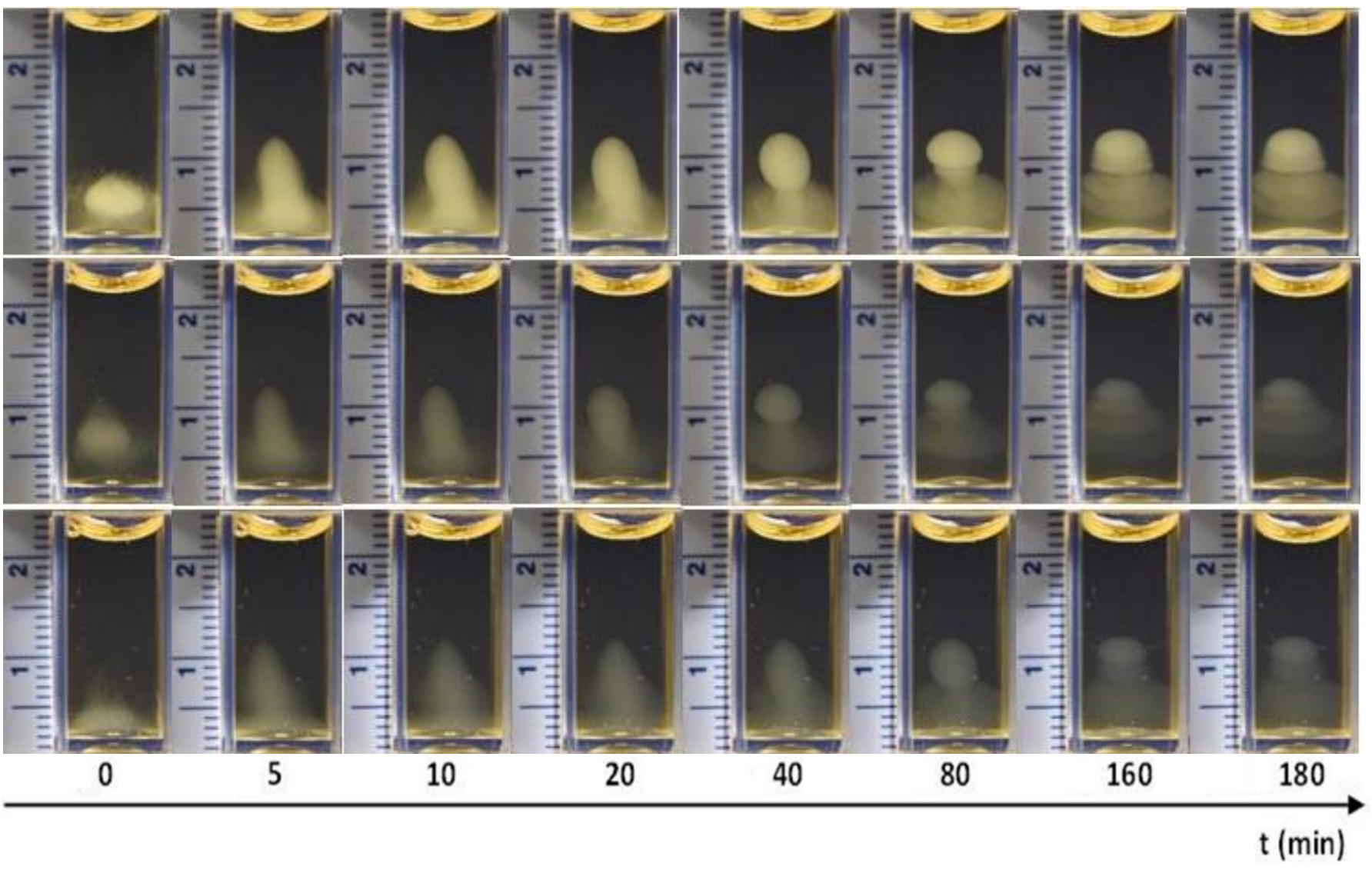

Figure S4: Photographs of the Levitation profiles of representative human plasma protein samples and formation of ellipsoidal patterns in MagLev at $0.06 \mathrm{mg} / \mathrm{ml}$ concentration of SPIONs. 


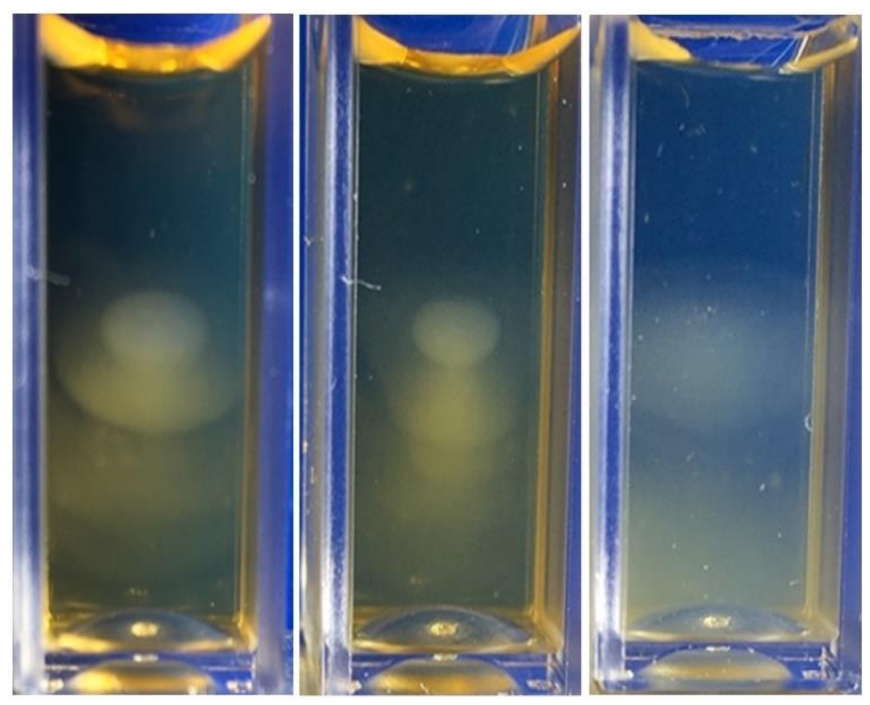

Figure S5: Levitation profiles of human plasma protein samples in the MagLev system at various concentrations of SPIONs (from left to right: $0.05 \mathrm{mg} / \mathrm{ml}, 0.03 \mathrm{mg} / \mathrm{ml}$, and $0.01 \mathrm{mg} / \mathrm{ml}$ ).

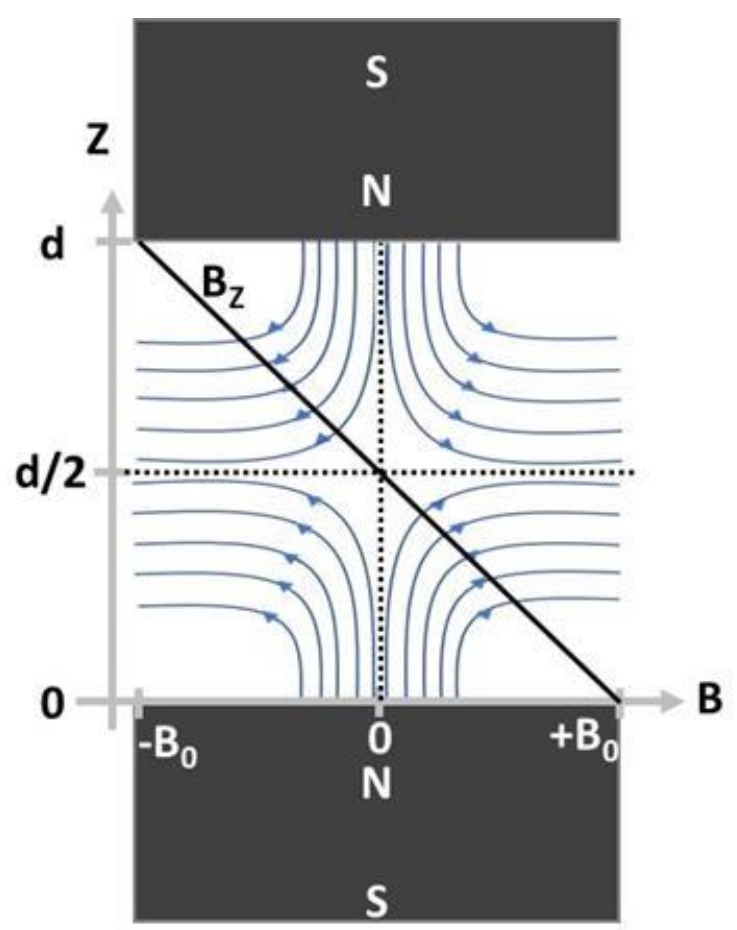

Figure S6: Levitation Schematic of magnetic field lines between two identical permanent magnets in the MagLev system separated by a distance of $\mathrm{d}$ and with like poles facing each other. Arrows shows the direction of the $\mathrm{B}$ field. The magnitude of the $\mathrm{z}$ component of the magnetic field vector, $B_{z}$, along the main symmetric axis of the MagLev is shown in the scheme. 


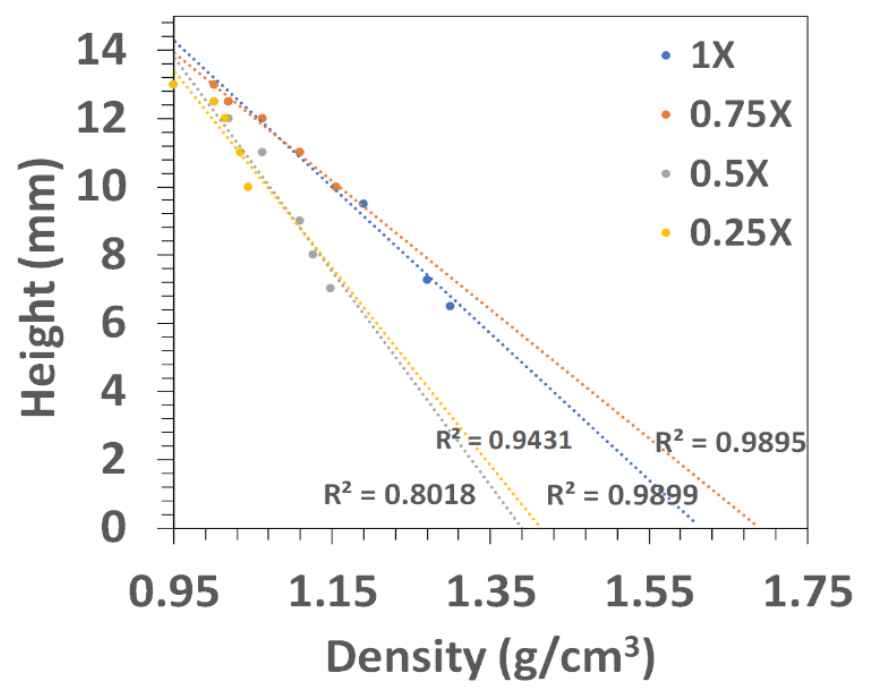

Figure S7: Calibration curves of the MagLev system using standard density glass particles and various concentrations of SPIONs $(0.25 X, 0.5 X, 0.75 X$ and $1 X, X=1 \mathrm{mg} / \mathrm{ml})$. The $\mathrm{R}^{2}$ values for the linear fits are depicted inside the diagram for each specific concentration of SPIONs.

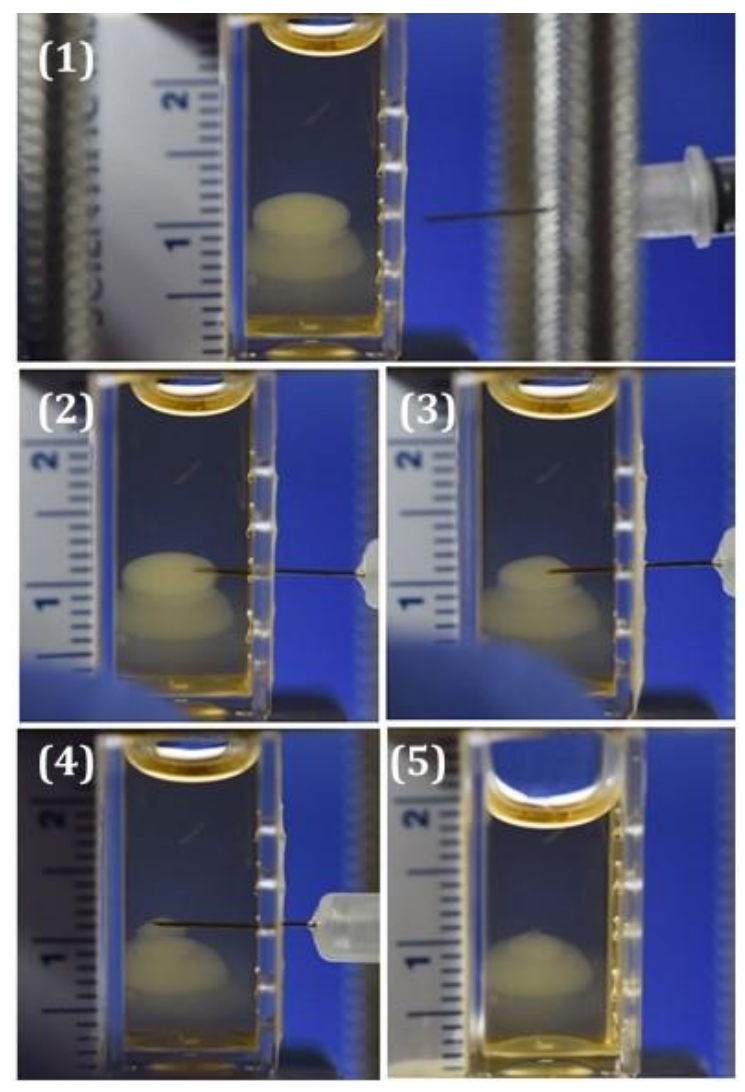

Figure S8: Typical extraction process of protein bands in the MagLev system: (1) before extraction, (2) beginning of extraction, (3) middle of the extraction process, (4) final stage of extraction and (5) end of extraction process for top band. 


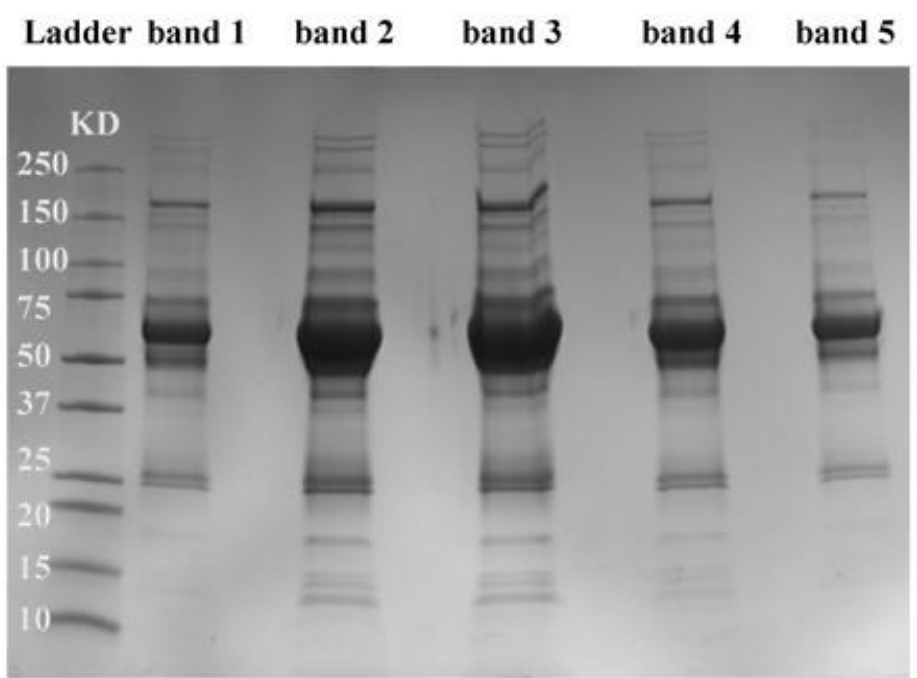

Figure S9: SDS-PAGE analysis of 5 different extracted bands (from top to bottom) from the MagLev system in addition to one ladder protein sample as a control. The concentrations of the SPIONs were $0.06 \mathrm{mg} / \mathrm{ml}$. 
ONLY PROTEINS OVER 2\% RETAINED

Protein Name

Complement $\mathrm{C} 3$

Alpha-2-macroglobulin

Hemopexin

Immunoglobulin kappa constant

Serotransferrin

Fibrinogen alpha chain

Haptoglobin-related protein

Inter-alpha-trypsin inhibitor heavy chain $\mathrm{H} 2$

Serum albumin OS=Homo sapiens

Haptoglobin

Immunoglobulin kappa constant

Fibrinogen gamma chain

Immunoglobulin heavy constant gamma 3

\begin{tabular}{|c|c|c|c|c|c|c|}
\hline MW (kD) & 1 (top) & 2 & 3 & 4 & 5 & 6 (bottom) \\
\hline 12.7 & 11.3 & 11.3 & 11.1 & 11.3 & 12.3 & 10.3 \\
\hline 12.9 & 8.6 & 5.8 & 6.4 & 6.7 & 6.0 & 6.2 \\
\hline 12.5 & 4.8 & 3.4 & 4.6 & 4.9 & 4.1 & 5.3 \\
\hline 10.8 & 4.6 & 5.2 & 4.9 & 4.9 & 4.8 & 4.1 \\
\hline 71.9 & 4.3 & 6.4 & 6.1 & 6.2 & 5.9 & 6.0 \\
\hline 44.7 & 3.8 & 1.3 & 1.1 & 1.0 & 2.8 & 3.1 \\
\hline 35.9 & 3.4 & 3.5 & 3.7 & 3.3 & 2.7 & 2.8 \\
\hline 12.8 & 3.1 & 1.7 & 2.5 & 2.2 & 2.2 & 2.5 \\
\hline 69.3 & 3.1 & 4.2 & 4.1 & 3.8 & 3.6 & 4.2 \\
\hline 39.3 & 3.1 & 3.8 & 3.6 & 3.5 & 3.4 & 3.6 \\
\hline 10.8 & 2.8 & 3.4 & 3.2 & 2.8 & 2.8 & 2.9 \\
\hline 42 & 2.5 & 0.3 & 0.2 & 0.2 & 1.2 & 1.7 \\
\hline 12.6 & 2.0 & 3.0 & 2.9 & 3.1 & 2.1 & 2.0 \\
\hline & 15.3 & 14.7 & 15.0 & 14.5 & 14.9 & 15.0 \\
\hline
\end{tabular}

Avg. MW (kD) of mixture

Figure S10: Liquid chromatography mass spectroscopy (LC MS/MS) outcomes of different extracted bands of the levitated plasma proteins. Numbers associated to proteins in each band demonstrates a participation percentage of each protein in the bands. The calculation of protein concentration according to the mass spectral counting $(\mathrm{SpC})$ method has been described elsewhere ${ }^{1,2}$. Full list of the identified proteins and their concentration in each bands is available in Excel file S1.

(a)

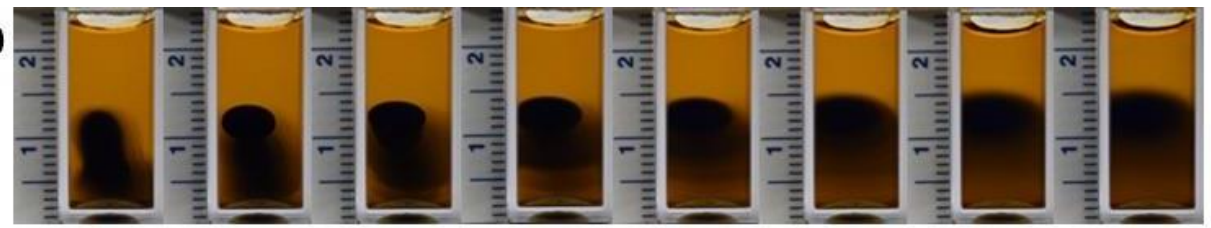

(b)

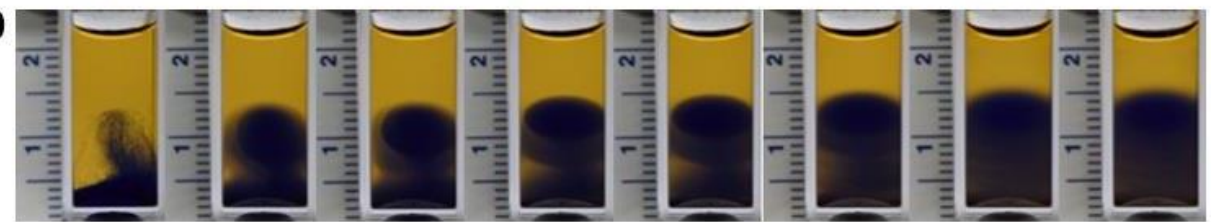

(c)

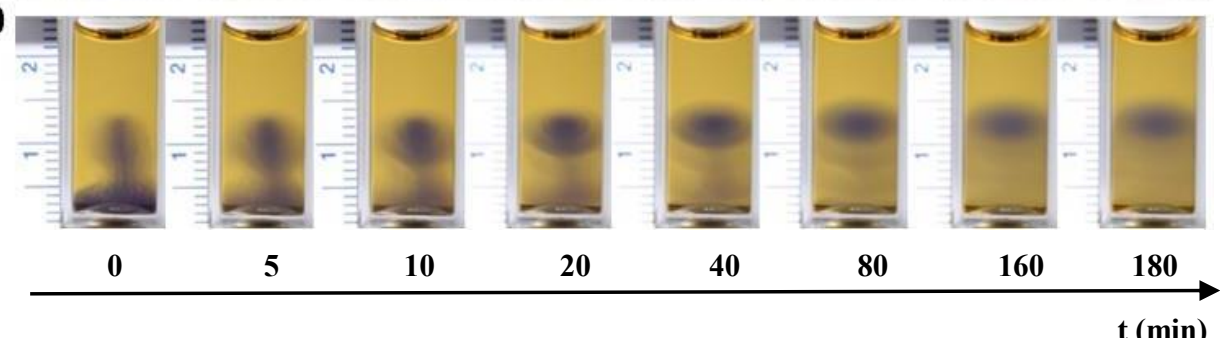

Figure S11: Levitation patterns of standard protein samples (ten-protein ladder ranging from 9 to $250 \mathrm{kD}$ ) in the MagLev platform at (a) $0.25 \mathrm{mg} / \mathrm{ml}$, (b) $0.125 \mathrm{mg} / \mathrm{ml}$ and (c) $0.06 \mathrm{mg} / \mathrm{ml}$ concentrations of SPIONs. 


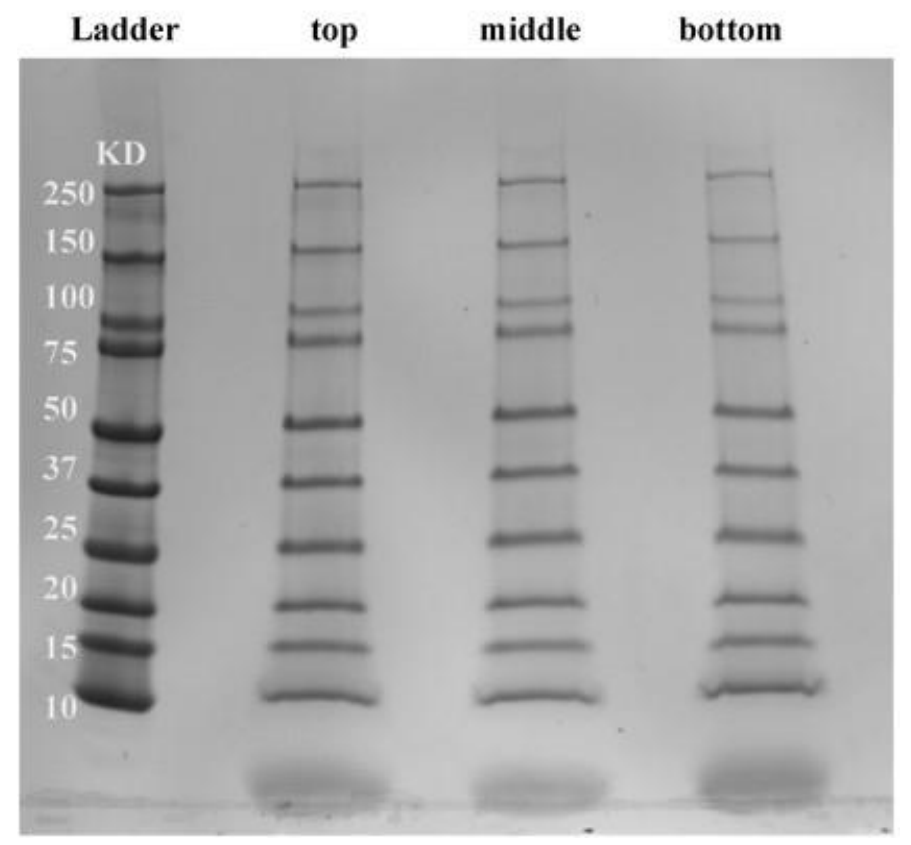

Figure S12: SDS-PAGE results of standard proteins extracted from top, middle and bottom appeared bands in the MagLev and 0.125 $\mathrm{mg} / \mathrm{ml}$ concentration of SPIONs.

\section{ONLY PROTEINS OVER 2\% RETAINED \\ Protein Name \\ Alpha-2-macroglobulin \\ Serum albumin \\ Complement C3 \\ Alpha-1-antichymotrypsin \\ Alpha-1-antitrypsin \\ Serotransferrin \\ Fibrinogen beta chain \\ Immunoglobulin heavy constant gamma 1 \\ Immunoglobulin heavy constant gamma 3 \\ Immunoglobulin kappa constant \\ Sum of the identified proteins in each band (\%)}

\begin{tabular}{|c|c|c|c|c|c|c|}
\hline \multicolumn{7}{|c|}{ Bands } \\
\hline MW (kD) & 1 (top) & 2 & 3 & 4 & 5 & 6 (bottom) \\
\hline 77 & 3.4 & 3.3 & 4.0 & 3.7 & 6.4 & 2.6 \\
\hline 69.3 & 38.3 & 38.2 & 45.8 & 43.2 & 58.4 & 69.6 \\
\hline 52.9 & 0.8 & 2.2 & 1.2 & 1.2 & 1.0 & 0.7 \\
\hline 47.6 & 1.7 & 2.5 & 2.2 & 0.0 & 0.0 & 0.0 \\
\hline 46.7 & 4.2 & 4.0 & 4.2 & 4.3 & 9.3 & 4.4 \\
\hline 39 & 4.0 & 3.1 & 2.7 & 2.7 & 2.6 & 2.2 \\
\hline 37.6 & 2.0 & 3.1 & 1.3 & 2.1 & 0.0 & 0.0 \\
\hline 36.1 & 5.8 & 6.6 & 7.1 & 19.5 & 10.1 & 9.2 \\
\hline 35.9 & 0.5 & 2.1 & 0.5 & 1.5 & 1.2 & 1.3 \\
\hline \multirow[t]{3}{*}{11.8} & 20.5 & 19.6 & 19.1 & 7.8 & 0.0 & 0.0 \\
\hline & 81.3 & 84.6 & 88.0 & 85.8 & 89.1 & 89.9 \\
\hline & 39.4 & 41.0 & 45.0 & 45.7 & 55.4 & 57.3 \\
\hline
\end{tabular}

Figure S13: Liquid chromatography mass spectroscopy (LC MS/MS) outcomes of different extracted bands of the levitated ladder proteins. Numbers associated to proteins in each band demonstrates a participation percentage of each protein in the bands. The calculation of protein concentration according to the mass spectral counting ( $\mathrm{SpC}$ ) method has been described elsewhere ${ }^{1,2}$. Full list of the identified proteins and their concentration in each band is available in Excel file S2. 


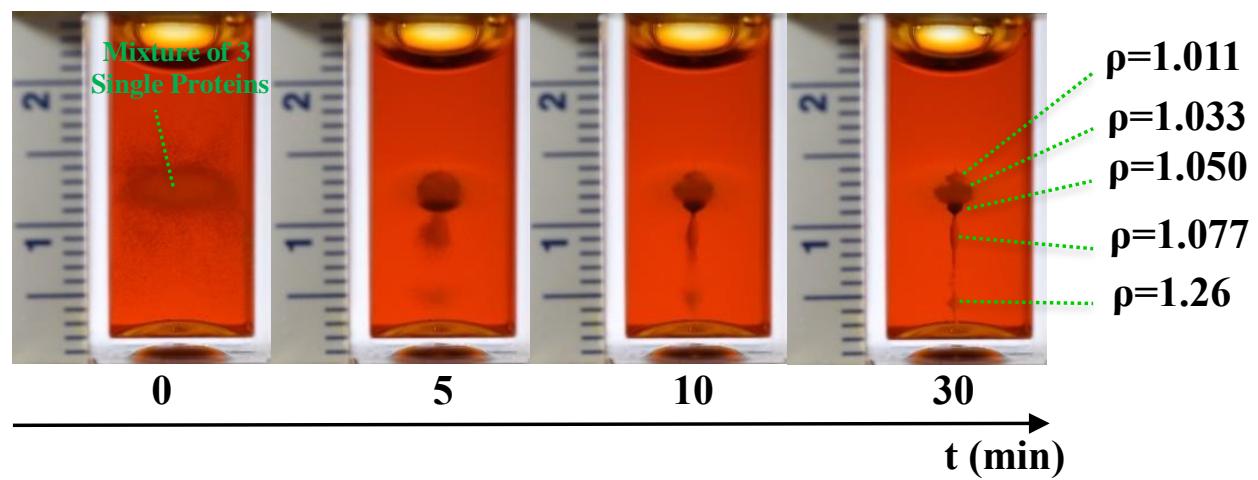

Figure S14: Levitation profiles of a mixture of three mixed different molecular weights of single proteins (Lysozyme, Albumin, and Immunoglobulin G (IgG)) with five standard density fluorescent polyethylene microspheres in the MagLev system (1 mg/ml concentration of SPIONs). All density units are $\mathrm{g} / \mathrm{cm}^{3}$.

\section{References}

1. M. Mahmoudi, S.E. Lohse, C. J. Murphy, A. Fathizadeh, A. Montazeri and K. Suslick, Nano Lett. 2014, 14, 6-12.

2. M. P. Monopoli, D. Walczyk, A. Campbell, G. Elia, I. Lynch, F. Baldelli Bombelli, K. A. Dawson, JACS 2011, 133, $2525-2534$. 